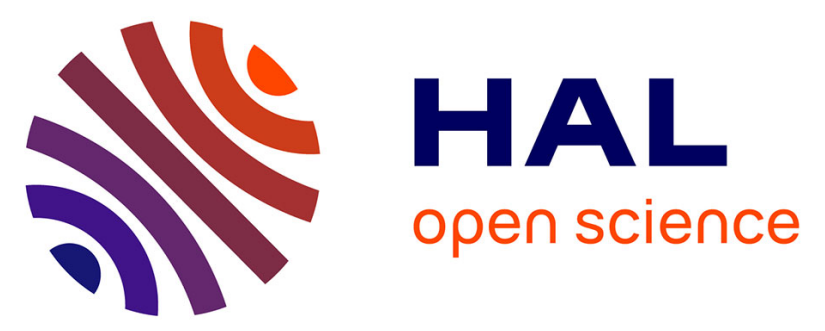

\title{
The combination of block copolymers and phospholipids to form Giant Hybrid Unilamellar Vesicles (GHUVs) does not systematically lead to "intermediate" membrane properties.
}

Tuyen P T Dao, Fabio Bras Fernandes, Martin Fauquignon, Emmanuel Ibarboure, Manuel Prieto, Jean-François F Le Meins

\section{To cite this version:}

Tuyen P T Dao, Fabio Bras Fernandes, Martin Fauquignon, Emmanuel Ibarboure, Manuel Prieto, et al.. The combination of block copolymers and phospholipids to form Giant Hybrid Unilamellar Vesicles (GHUVs) does not systematically lead to "intermediate" membrane properties.. Soft Matter, 2018, 14 (31), pp.6476-6484. 10.1039/C8SM00547H . hal-01846474v2

HAL Id: hal-01846474

https://hal.science/hal-01846474v2

Submitted on 18 Nov 2018

HAL is a multi-disciplinary open access archive for the deposit and dissemination of scientific research documents, whether they are published or not. The documents may come from teaching and research institutions in France or abroad, or from public or private research centers.
L'archive ouverte pluridisciplinaire HAL, est destinée au dépôt et à la diffusion de documents scientifiques de niveau recherche, publiés ou non, émanant des établissements d'enseignement et de recherche français ou étrangers, des laboratoires publics ou privés. 
Received 16th March 2018, Accepted 11th July 2018

DOI: $\underline{10.1039 / 10.1039 / C 8 S M 00547 H ~}$

https://hal.archivesouvertes.fr/hal-01846474

\title{
The combination of block copolymers and phospholipids to form Giant Hybrid Unilamellar Vesicles (GHUVs) does not systematically lead to "intermediate" membrane properties.
}

\author{
T.P.T. Dao ${ }^{1,2,3}$, F. Fernandes $*^{3}$, M. Fauquignon ${ }^{1,2}$, E. Ibarboure ${ }^{1,2}$, M. Prieto $^{3}$, and J. F. Le Meins ${ }^{1,2}$
}

In this work, the elasticity under stretching as well as fluidity of Giant Hybrid Unilamellar Vesicles (GHUV) has been studied. The membrane structuration of these GHUVs has been already studied at micro and nanoscale in a previous study of the team ${ }^{1}$. These GHUVs were obtained by the association of fluid phospholipid (POPC) and triblock copolymer poly(ethyleneoxide)-b-poly(dimethylsiloxane)-b-poly(ethyleneoxide). Although triblock copolymers' architecture can facilitate vesicle formation, they have been scarcely used to generate GHUVs. We show, through micropipette aspiration and FRAP experiments, that the incorporation of a low amount of lipids in the polymer membrane leads to a significant loss of the toughness of the vesicle and subtle modification of lateral diffusion of polymer chains. We discuss the results within the framework of conformation of the triblock copolymer chain in the membrane, and of the presence of lipid nanodomains.

\section{Introduction}

Hybrid polymer/lipid vesicles are the subject of an increasing number of studies by different scientific communities (bioengineers, chemists, physical chemists, pharmacists, biochemists). The driving force behind this surging interest is the design of entities that could combine all the benefits of each component (biofunctionality, permeability of lipid phases, mechanical stability, chemical versatility of polymer phase...). These emerging systems have already been the subject of two reviews. ${ }^{2,3}$ A significant number of studies have focused on their use in different fields, such as drug delivery ${ }^{4-7}$, drug targeting, ${ }^{8}$ nanoreactors, ${ }^{7,9,10}$ biomolecular recognition and interaction with nanoparticles ${ }^{11-14}$, or their interaction with biological media. ${ }^{15}$ So far, only a relatively moderate part of the work published on the subject has focused on the selfassembly problematic: how can we modulate the phase separation process during the self-assembly, preventing formation of separated liposomes and polymersomes, and obtain membranes with lipid domains of controlled size? The relation-ship between membrane composition (\% polymer lipid, presence of domains) and properties like elasticity, permeability, tumor-targeting ability, is not clearly established so far, especially at the nanoscale in Large Hybrid Unilamellar Vesicles, LHUV, often used in the above mentioned studies. Indeed, proof of hybrid character and information about membrane structuration are not easy to obtain at nanoscale.

\footnotetext{
a. University of Bordeaux, LCPO UMR 5629, 16 avenue Pey Berland, F-33600 Pessac, France;

${ }^{b}$ CNRS, Laboratoire de Chimie des Polymères Organiques, UMR 5629, F-33600, Pessac, France

CQFM-IN and IBB-Institute for Bioengineering and Biosciences, Instituto Superior Técnico, Universidade de Lisboa, Lisboa, Portugal.

*: corresponding author : lemeins@enscbp.fr, fernandesf@tecnico.ulisboa.pt

Electronic Supplementary Information (ESI) available: [Membrane mechanical properties, Micropipette aspiration and FRAP experiments.) See DOI: $10.1039 / \mathrm{C} 8 \mathrm{SM} 00547 \mathrm{H}$
}

DLS and $\mathrm{DSC}^{4}$ have been used to evaluate the efficiency of polymer/lipid mixing but a real quantification is very hard to reach. Moreover, different structures can be obtained (e.g.; wormlike hybrid micelles, or tubular vesicles), depending on the molar mass of the copolymer and lipid fractions. The presence of such heterogeneous structures, most of the time revealed by CryoTEM analysis ${ }^{9,16-18,}$ can limit the optimization of the benefits/advantages observed in different applications (e.g. drug delivery). Time resolved fluorescence spectroscopy techniques have been shown to be very useful to probe the efficiency of polymer/lipid mixing at the nanoscale. ${ }^{17}$ Especially, dual-colour fluorescence cross-correlation spectroscopy (DC-FCCS) appears to be an efficient and elegant way to prove and quantify the hybrid character of vesicles at the nanoscale ${ }^{19}$. Fluorescence Lifetime Imaging technique (FLIM) is also a powerful approach to probe membrane structuration in GHUVs. ${ }^{1}$

So far, most of the existing studies focusing on the understanding of parameters governing membrane structuration have been made on giant vesicles obtained by electroformation. ${ }^{1,20-24}$ Information has also been obtained through evaluation of nanoparticle-membrane interaction. ${ }^{11}$ Globally, hydrophobic mismatch resulting from the difference of thickness between polymer and lipid hydrophobic cores, as well as lipid fluidity, have been identified as important parameters in membrane structuration. The cooling process after formation of giant vesicles at temperatures above the melting point of phospholipids, is also of paramount importance. ${ }^{1,20,24}$

Scarce information is available regarding the mechanical properties of these hybrid vesicles. Area expansion modulus (or stretching modulus), lysis strain and stress have been estimated for two systems PBut-b-PEO/POPC ${ }^{21}$ and PDMS-gPEO/DPPC ${ }^{24}$, in the presence of a specific molar fraction of lipid (30\%). In the case of PBut-b-PEO, values for the stretching modulus were found to be intermediate between pure lipid 
This document is the Accepted Manuscript version of an article that appeared in final form in Soft Matter, 2018, 14(31), 6476-6484, after peer review and technical editing by the publisher. To access the final edited and published work see DOI: 10.1039/C8SM00547H

and pure polymer membranes (e.g. $17 \%$ higher for hybrid vesicles than that of pure polymersomes), whereas the effect was far less pronounced for PDMS-g-PEO/DPPC vesicles (7\% higher stretching modulus for hybrid vesicles), which present patchy domains of DPPC for such fraction. Regarding membrane fluidity, the mobility of lipids has been evaluated by Fluorescence Recovery After Photobleaching (FRAP) measurements in PBut-b-PEO/POPC ${ }^{21}$ and PIB-b-PEO/DPPC GHUVs $^{13}$ and has been shown to be modulated by the amount of polymer. However their diffusion within the membrane seems to be complex in PBut-b-PEO/POPC ${ }^{21}$ hybrid vesicles, and cannot be described by a standard lateral diffusion model with one diffusion coefficient.

Recently, our group has performed a systematic study aiming at revealing the influence of hydrophobic mismatch on the membrane structuration of GHUV by mixing different triblock copolymer PEO-b-PDMS-b-PEO of different molar masses with POPC or DPPC. ${ }^{1}$ The triblock architecture in our previous studies was chosen as it favours formation of a vesicular structure in a larger range of hydrophilic fractions compared to diblock. Depending on the copolymer molar mass, a homogenous or a heterogenous membrane at the microscale can be obtained, likely as a result of different line tensions at the lipid/polymer boundaries. Interestingly, lipid nanodomains have been detected by Förster Resonance Energy Transfer (FRET) imaging measurements carried out through FLIM. Membrane elastic properties of giant vesicles obtained from the self-assembly of triblock copolymers are unknown, and so far data are only available for diblock, ${ }^{25-29}$ or grafted copolymers $^{30}$. In addition, the membrane properties resulting from their association with fluid phospholipids are also unknown. It is interesting to note that differences in terms of membrane fluidity between diblock and triblock copolymers have recently been shown in a systematic FCS study performed on Giant Unilamellar Vesicle (GUV). ${ }^{31}$ It was suggested that triblock copolymers adapt mixed conformations between an extended shape and a $U$-shape that may reduce their mobility. In this work, we propose to analyse and discuss the elastic properties and membrane fluidity of Hybrid Giant Unilamellar Vesicles resulting from self-assembly of triblock copolymers and POPC. Micropipette aspiration technique (MPA), ${ }^{32,33}$ and FRAP measurements were carried out with that purpose. Two different molar mass of PDMS were considered, as this parameter is of importance regarding the structuration of hybrid membrane as mentioned previously. In addition, the influence of copolymer architecture on the polymersome's membrane properties will be shortly discussed.

\section{Materials and methods}

\section{Materials}

1-Palmitoyl-2-oleoyl-sn-glycero-3-phosphocholine (POPC) and 1,2-dioleoyl-sn-glycero-3-phosphoethanolamine- $\mathrm{N}$-(lissamine rhodamine B sulfonyl) (DOPE-Rhod) were obtained from Avanti Polar Lipids Inc., and used without further purification.
Amphiphilic graft copolymer PDMS ${ }_{26}-\mathrm{g}-\left(\mathrm{PEO}_{12}\right)_{2}$ was a gift from Dow Corning Inc. (\#5329) and thereafter denoted DOW. The characterization of this copolymer was performed in the lab in a previous study ${ }^{34}$, and all the results were in agreement with data given by the manufacturer and data available in literature ${ }^{35,36}$ This copolymer forms vesicles with a membrane thickness close to $5 \mathrm{~nm}$. We used this copolymer to validate our MPA procedure, as GUVs of this copolymer have already been studied by MPA and their stretching elasticity is well established. Fluorescein-labelled graft copolymer $\left(\mathrm{PDMS}_{26^{-}}\right.$-g$\left.\left(\mathrm{PEO}_{12}\right)_{2}-\mathrm{F}\right)$ was prepared as previously described. ${ }^{23}$ Triblock copolymers have been synthesised following a procedure described in previous studies ${ }^{1}$. Their molecular characteristics are recalled in the Table 1.

\begin{tabular}{|c|c|c|c|c|}
\hline Name & Composition & $\begin{array}{c}\mathrm{Mn} \\
(\mathrm{g} / \mathrm{mol}) \\
(1 \mathrm{H} \text { NMR) }\end{array}$ & 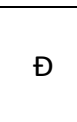 & $\begin{array}{c}d \\
(\mathrm{~nm})\end{array}$ \\
\hline DOW & $\mathrm{PDMS}_{26} \mathrm{~g}^{\mathrm{g}}\left(\mathrm{PEO}_{12}\right)_{2}$ & 2750 & 1.32 & $5.6 \pm 0.6^{*}$ \\
\hline $1.5 \mathrm{~K}$ & $\begin{array}{c}\mathrm{PEO}_{8}-\mathrm{b}-\mathrm{PDMS}_{22}-\mathrm{b}- \\
\mathrm{PEO}_{8}\end{array}$ & 2668 & 1.18 & $5.4 \pm 0.4$ \\
\hline $3 \mathrm{~K}$ & $\begin{array}{c}\text { PEO }_{12}-\mathrm{b}-\mathrm{PDMS}_{43}-\mathrm{b}- \\
\mathrm{PEO}_{12}\end{array}$ & 5014 & 1.28 & $8.8 \pm 0.5$ \\
\hline
\end{tabular}

Table 1. Molecular characteristics of amphiphilic copolymers used in this study, and corresponding membrane thickness $d$, from $^{1}{ }^{*}$ from ${ }^{23}$

\section{Generation of GUVs}

GUVs were generated by the electro-formation method, which is based on the process reported by Angelova. ${ }^{37}$ Coverslips were cleaned with acetone and ethanol. The initial lipid or polymer solutions were prepared in a chloroform/methanol mixture $(2 / 1 \mathrm{vol} / \mathrm{vol})$ at a concentration of $1 \mathrm{mM}$. For FRAP and MPA experiments, DOPE-Rhod was included at $0.2 \mathrm{~mol} \%$ of lipid content, while Dow-F was included at $1.5 \mathrm{~mol} \%$ of polymer content.

For electro formation, about $3 \mu \mathrm{L}$ of the solution was spread slowly on both sides of Pt electrodes. ITO plates (resistivity 15$35 \Omega . \mathrm{cm}$ ) were also used. After evaporation of the solvent during at least 5-6 hours, the electrodes were connected to AC voltage (sinusoidal tension $2 \mathrm{~V}, 10 \mathrm{~Hz}$ ) and submerged in a 100 $\mathrm{mM}$ sucrose solution. Electro formation was performed at room temperature both for POPC or triblock copolymers. Samples were collected after 75 minutes and stored at room temperature, in the dark for samples containing fluorescent analogues, until measurements.

\section{Confocal fluorescence microscopy}

Confocal fluorescence microscopy was performed on a Leica TCS SP5 (Leica Microsystems CMS GmbH, Mannheim, Germany) inverted confocal microscope (DMI6000). A 63x apochromatic water immersion objective with a NA of 1.2 (Zeiss, Jena Germany) was used for all experiments. Dow-F and DOPE-Rhod excitation were achieved with the $488 \mathrm{~nm}$ and 514 $\mathrm{nm}$ argon laser lines respectively, while fluorescence emission 
This document is the Accepted Manuscript version of an article that appeared in final form in Soft Matter, 2018, 14(31), 6476-6484, after peer review and technical editing by the publisher. To access the final edited and published work see DOI: 10.1039/C8SM00547H

was collected in the 500-530 $\mathrm{nm}$ range for Dow-F and in the 600-700 $\mathrm{nm}$ range for DOPE-Rhod.

\section{Fluorescence recovery after photobleaching}

Membrane fluidity (or viscosity) has an impact on both molecular rotational, and translational diffusion rates, described through the translational diffusion coefficient (D). In this section, the translational fluidity of the membrane is reported via Fluorescence recovery after photobleaching (FRAP) measurements. The phospholipid analogue DOPE-Rhod was used to study the mobility of phospholipids, whereas fluorescein modified grafted copolymer Dow-F was used to evaluate mobility of polymer chains. Investigations on both pure liposome and polymersome were performed in a first step in order to obtain reference values and validate all setting and experimental procedures. For the FRAP experiments, the same confocal microscope setup was used, using the above described conditions for excitation/emission. FRAP measurements of Dow- $F$ were started with 10 image scans at low laser intensity $(488 \mathrm{~nm})$. Then Fluorescein was photobleached inside a region of interest (ROI) sized $5 \mu \mathrm{m}$, using a scan of 3 frames at high laser intensity (488 nm). Finally, the fluorescence recovery was monitored by the acquisition of a series of 150 images with the same low laser intensity as the pre-bleach. In all steps, the images were acquired using a frame size of $256 \times 256$ pixels and bidirectional scanning at a $1400 \mathrm{~Hz}$ line frequency scan speed, which gave a time-lapse of $113 \mathrm{~ms}$ per image. All measurements were made at $\mathrm{T}=20^{\circ} \mathrm{C} \pm 1^{\circ} \mathrm{C}$.

To fully immobilize the vesicles during acquisition, biotinylated lipid $\left(10^{-4} \mathrm{~mol} \%\right)$ was added in the initial lipid mixture. Vesicles obtained were transferred to the wells of an eight-well $\mu$-Slide from Ibidi (Munich, Germany) previously coated with avidin, 15 minutes before measurement. The avidin coating was carried out through incubation of $200 \mu \mathrm{L}$ of an avidin solution at $0.1 \mathrm{mg} / \mathrm{ml}$ during at least $1 \mathrm{~h}$ at $4^{\circ} \mathrm{C}$ and subsequently washed with MilliQ-water to remove all the excess avidin. The FRAP data was first normalized to take into account the variations in the absolute amount of fluorescent molecules between samples. In this way, the relative fluorescence changes after photobleaching become proportional to the initial values and independent of fluorophore concentration. The method used in this work was double normalization:

$$
I_{\text {Norm }}(t)=\frac{I_{\text {Ref_pre }}}{I_{\text {Ref }}(t)-I_{\text {Bck }}(t)} \cdot \frac{I_{\text {Frap }}(t)-I_{\text {Bck }}(t)}{I_{\text {Frap pre }}}
$$

where $I_{\text {Norm }}(t)$ : normalized intensity, $I_{\text {Frap }}(t)$ : measured average intensity inside the bleached spot, $I_{\text {Ref }}(t)$ : measured average reference intensity and $I_{\mathrm{Bck}}(\mathrm{t})$ : measured average background intensity outside the ROI (Region Of Interest). Subscript _pre means the averaging of intensity in the corresponding ROI, immediately before bleaching and after subtraction of background intensity.
All experimental data was fitted with the circular spot model in 2D diffusion, described by:

$$
\begin{aligned}
I_{\text {Norm }}(t)= & a_{0}+a_{1} \cdot e^{\frac{\tau}{2\left(t-t_{\text {bleach }}\right)}} \cdot\left(I_{0}\left(\frac{\tau}{2\left(t-t_{\text {bleach }}\right)}\right)\right. \\
& \left.+I_{1}\left(\frac{\tau}{2\left(t-t_{\text {bleach }}\right)}\right)\right) \quad \text { (Eq. 2) }
\end{aligned}
$$

where $a_{0}$ and $a_{1}$ are constants and $t_{\text {bleach }}$ the characteristic time for diffusion. $I_{0}$ and $I_{1}$ are the modified Bessel function of order 0 and 1.

Analysis of FRAP data was performed using the FRAP analyser software version 1.0.5 (University of Luxembourg, Cytoskeleton and Cell Plasticity Laboratory, http://actinsim.uni.lu/).

The mobile fraction was also evaluated, to determine the presence of immobile fluorescent molecules that cannot participate in the exchange between bleached and unbleached regions, resulting in an incomplete recovery of the fluorescence signal. The mobile fraction is defined by:

$$
\mathrm{M}_{\mathrm{f}}=1-\mathrm{IM}_{\mathrm{f}}
$$

With

$$
\mathrm{IM}_{\mathrm{f}}=\frac{\mathrm{I}_{\mathrm{i}}-\mathrm{I}_{\infty}}{\mathrm{I}_{\mathrm{i}}-\mathrm{I}_{0}}
$$

$I \mathrm{M}_{\mathrm{f}}$ is referred as the immobile fraction and $\mathrm{I}_{\mathrm{i}}, \mathrm{I}_{0}$ and $\mathrm{I} \infty$ are the fluorescence intensity at initial pre-bleached time, bleached time and at maximal recovery respectively.

\section{Micropipette aspiration}

Micropipette aspiration technique was carried out also on a confocal microscope. The micropipettes were obtained by stretching borosilicate capillaries (1mmOD, $0.58 \mathrm{mmID})$ from WPI, with a pipette puller (Sutter Instrument P-97). The pulled pipets were then forged to the desired diameter $(6-8 \mu \mathrm{m})$ using a micro-forge Narishige MF-900. Before using, micropipettes were coated with BSA to prevent vesicle adhesion, by suction via the pipette of a filtrated BSA solution at $0.1 \%$. Thereafter, the pipettes were filled with appropriate solution ( $0.1 \mathrm{M}$ sucrose, water...) and connected via flexible tubing to a micromanipulator (Eppendorf, Patchman NP2). The vesicle tension was controlled by a home-made hydraulic watertight setup. The suction pressure exerted over the membrane is calculated from Equation 5:

$$
\mathrm{DP}=\left(\mathrm{h}-\mathrm{h}_{0}\right) \rho_{\mathrm{w}} \mathrm{g}
$$


This document is the Accepted Manuscript version of an article that appeared in final form in Soft Matter, 2018, 14(31), 6476-6484, after peer review and technical editing by the publisher. To access the final edited and published work see DOI: 10.1039/C8SM00547H

where $\rho_{\mathrm{w}}$ is the density water $\left(\rho=1 \mathrm{~g} \cdot \mathrm{cm}^{-3}\right), \mathrm{g}$ is the gravitational acceleration $\left(9.8 \mathrm{~m} \cdot \mathrm{s}^{-2}\right), \mathrm{h}$ is the position of the water reservoir and $h_{0}$ is the initial position where the pressure is zero.

The membrane tension was calculated classically from the Laplace equation:

$$
\sigma=\frac{P_{s} \times R_{p}}{2\left(1-\frac{R_{p}}{R_{v}}\right)}
$$

where $R_{p}$ and $R_{v}$ are the micropipette and vesicle radius (outside the micropipette), and $P_{S}$ is the suction pressure on the micropipette. A relative area change of the membrane $(\alpha)$ is defined as:

$$
\alpha=\frac{A-A_{0}}{A_{0}}
$$

$A_{0}$ being the membrane area of the vesicle at the lower suction pressure. $\alpha$ can be estimated from the increase in projection length $\Delta \mathrm{L}$ of vesicle inside the capillary tip according to Eq.8:

$$
\alpha=\frac{1}{2} \times \frac{\mathrm{R}_{\mathrm{p}}}{\mathrm{R}_{\mathrm{v}}^{2}} \times \Delta \mathrm{L} \times\left(1-\frac{\mathrm{R}_{\mathrm{p}}}{\mathrm{R}_{\mathrm{v}}}\right)
$$

Under very low tension regime, the apparent expansion is dominated by smoothing of thermal bending undulations. Plotting $\ln (\sigma)$ vs. $\alpha$ at low $\sigma$ values (typically $0.001-0.5 \mathrm{mN} \cdot \mathrm{m}^{-1}$ ${ }^{(32)}$, gives a straight line whose slope is related to the bending modulus, $\kappa$ (Eq.9). Under higher tensions, which were investigated in this work, (> $\left.0.5 \mathrm{mN} . \mathrm{m}^{-1}\right)$, membrane undulations are totally suppressed and membrane area increases as the result of increased spacing between molecules. In this regime, the rate of membrane area increase with tension can be used to calculate stretching or area expansion modulus Ka according to Eq.9. ${ }^{38}$ In the investigated tension regime, bending contribution is neglected and only the second term is considered to estimate Ka.

$$
\alpha \approx \frac{\mathrm{k}_{\mathrm{B}} \mathrm{T}}{8 \pi \kappa} \ln (\sigma)+\frac{\sigma}{\mathrm{K}_{\mathrm{a}}}
$$

Each parameter measured in this work is taken from an average value of at least 15 vesicles unless specified. The vesicles were analysed one day after electro formation.

\section{RESULTS AND DISCUSSION}

\section{Pure Polymersomes and Liposomes}

In order to validate our micropipette aspiration experimental procedure, measurement of stretching elasticity and lysis strain were performed on polymersomes obtained from selfassembly of grafted copolymer based on Poly(dimethylsiloxane) and poly(ethyleneoxide) Dow. The stretching elasticity of such membrane has already been studied previously, ${ }^{24,30}$ and this polymer has also been associated with phospholipids to generate GHUVs ${ }^{23,24}$ or LHUVs $^{39}$. Therefore this copolymer can be used as a reference.
The applied tension versus areal strain for the different copolymer used in this study is represented in Figure 1. From the slope the area expansion modulus is determined, and the values are indicated in Table 2. Histograms of Ka values for Dow and $3 \mathrm{~K}$ are available in ESI. (Fig S1 and S2). Considering the Dow GUVs, the area expansion modulus as well as lysis strain $\left(\alpha_{c}\right)$ and stress $\left(\sigma_{c}\right)$ are very similar to those reported in literature $\left(\sigma_{\mathrm{c}^{\sim}} 92 \pm 5 \mathrm{mN} / \mathrm{m}, \alpha_{\mathrm{c}} \sim 8 \%\right)^{30}$ and confirm the robustness of our protocol. Measurements were performed on pure polymersomes made from $3 \mathrm{~K}$ triblock copolymers, and the recovered area expansion modulus $(84 \pm 13 \mathrm{mN} / \mathrm{m})$ is only slightly higher than the one obtained for $1.5 \mathrm{~K}(69 \pm 9 \mathrm{mN} / \mathrm{m})$, which has a lower molar mass and whose membrane mechanical properties has been evaluated in a previous study. ${ }^{40}$ The amplitude of the area expansion modulus is normally linked to the interfacial tension between blocks, and is not modulated by their molar masses. ${ }^{28,41}$ However, the lysis strain is more important for the $3 \mathrm{~K}$, and reflects an increase of the membrane toughness with the molar mass as already proven in a previous study. ${ }^{41}$ It is interesting to note that although these experiments are known to be complex to carry out, and are known to generate values with a relatively high uncertainty, a slight difference between the grafted copolymer and triblock copolymer seems to be observed, and suggests an influence of the copolymer architecture on the stretching elasticity of the membrane. This has to be confirmed in further studies.

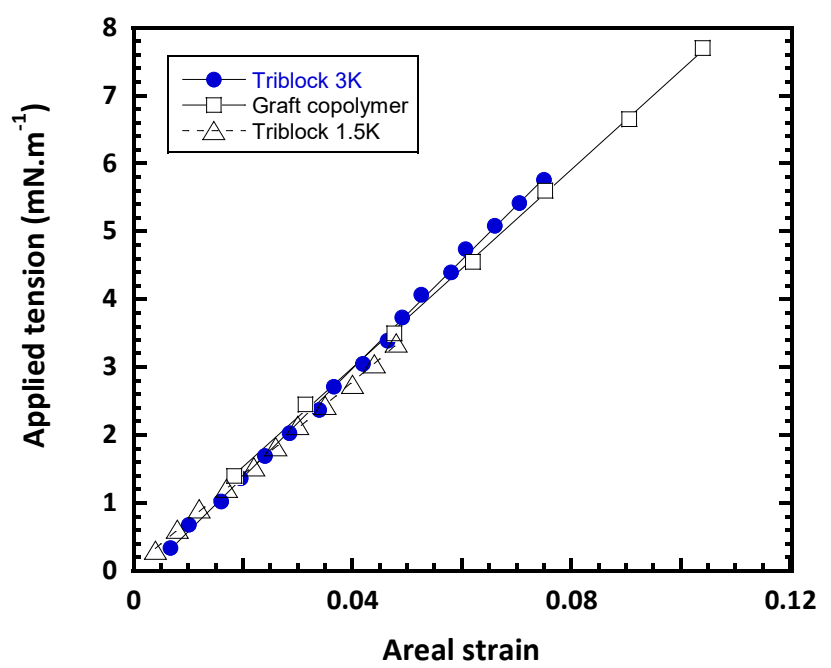

Figure 1: Tension versus areal strain for pure polymersomes. Data for $1.5 \mathrm{~K}$ copolymer were extracted from a previous study of our group. 
This document is the Accepted Manuscript version of an article that appeared in final form in Soft Matter, 2018, 14(31), 6476-6484, after peer review and technical editing by the publisher. To access the final edited and published work see DOI: 10.1039/C8SM00547H

\begin{tabular}{|c|c|c|c|c|c|c|c|c|c|c|}
\hline Polymer & $\begin{array}{l}\text { Stretching } \\
\text { Modulus } \mathrm{K}_{\mathrm{a}} \\
\left(\mathrm{mN} \cdot \mathrm{m}^{-1}\right)\end{array}$ & $\begin{array}{l}\text { Lysis Strain (\%) } \\
\qquad \alpha_{c}\end{array}$ & $\begin{array}{l}\text { Lysis stress } \\
\left(\mathrm{mN} \cdot \mathrm{m}^{-1}\right) \sigma_{\mathrm{c}}\end{array}$ & \multirow[t]{2}{*}{ Copolymer/Lipid } & \multirow[t]{2}{*}{$\begin{array}{l}\text { MPDMs } \\
\text { (g.mol } \\
{ }^{1} \text { ) }\end{array}$} & \multirow[t]{2}{*}{$\begin{array}{c}d \pm \\
\text { SD } \\
(\mathrm{nm})\end{array}$} & \multirow[t]{2}{*}{$\begin{array}{c}D \pm S D \\
\left(\mu m^{2} \cdot s^{-}\right. \\
\left.{ }^{1}\right)\end{array}$} & \multirow[t]{2}{*}{$\begin{array}{l}M_{f} \pm \\
S D\end{array}$} & \multirow[t]{2}{*}{$\begin{array}{c}\eta^{a} \\
\text { (Pa.s) }\end{array}$} & \multirow[t]{2}{*}{$\begin{array}{c}\eta^{\mathrm{b}} \\
\text { (Pa.s) }\end{array}$} \\
\hline DOW & $84 \pm 12$ & $8.1 \pm 0.9$ & $6.2 \pm 0.5$ & & & & & & & \\
\hline $\begin{array}{c}1.5 \mathrm{~K} \\
\left(\text { data from }^{40}\right)\end{array}$ & $69 \pm 9$ & $2.4 \pm 0.5$ & $2.7 \pm 0.7$ & \multirow[t]{2}{*}{ DOW } & \multirow[t]{2}{*}{1924} & \multirow[t]{2}{*}{$\begin{array}{c}5.6 \pm \\
0.6\end{array}$} & \multirow[t]{2}{*}{$\begin{array}{c}4.1 \pm \\
0.9\end{array}$} & $\begin{array}{c}0.95 \\
\pm\end{array}$ & \multirow[t]{2}{*}{0.094} & \multirow[t]{2}{*}{0.077} \\
\hline $3 \mathrm{~K}$ & $84 \pm 13$ & $7.3 \pm 1.3$ & $5.5 \pm 1.7$ & & & & & $\begin{array}{l}0.07 \\
1.00\end{array}$ & & \\
\hline \multicolumn{4}{|c|}{$\begin{array}{l}\text { Table 2: Membrane mechanical characteristics of the different polymersomes used in } \\
\text { this study. }\end{array}$} & $1.5 \mathrm{~K}$ & 1628 & $\begin{array}{c}5.4 \pm \\
0.4\end{array}$ & $\begin{array}{c}3.7 \pm \\
0.8\end{array}$ & $\begin{array}{c} \pm \\
\pm \\
0.01\end{array}$ & 0.108 & 0.102 \\
\hline \multirow{2}{*}{\multicolumn{4}{|c|}{$\begin{array}{l}\text { The diffusion coefficient in polymersomes and liposomes } \\
\text { membrane has been also measured by FRAP, following the } \\
\text { procedure described previously. The results are summarized in } \\
\text { Table 3. FRAP curves can be consulted in supporting } \\
\text { information. (Fig S3) }\end{array}$}} & $3 \mathrm{~K}$ & 3182 & $\begin{array}{c}8.8 \pm \\
0.5\end{array}$ & $\begin{array}{c}1.9 \pm \\
0.6\end{array}$ & $\begin{array}{c}0.97 \\
\pm \\
0.04\end{array}$ & 0.130 & 0.132 \\
\hline & & & & POPC & - & $4.0^{*}$ & $\begin{array}{c}9.9 \pm \\
1.6\end{array}$ & $\begin{array}{c}1.00 \\
\pm \\
0.02\end{array}$ & - & $0.044^{* *}$ \\
\hline
\end{tabular}

Following Saffman Delbück's continuum hydrodynamic model

42 describing lateral and rotational diffusion of cylindrical objects moving in a two dimension fluid (e.g. a lipid membrane), the lateral diffusion coefficient (D) can be expressed as:

$$
\mathrm{D}=\frac{\mathrm{k}_{\mathrm{b}} \mathrm{T}}{4 \pi \eta \mathrm{h}}\left(\ln \left(\frac{\eta \mathrm{h}}{\eta^{\prime} \mathrm{R}}\right)-\gamma\right)
$$

where $k_{b}$ is the Boltzmann constant, $T$ is absolute temperature, $h$ is the thickness of the bilayer, $\eta$ is viscosity of the membrane, $\eta^{\prime}$ is viscosity of the outer liquid, $R$ is the radius of the diffusing object and $\gamma$ is Euler's constant. Using this equation, the membrane viscosities were calculated from our measurement of diffusion coefficient. The hydrophobic thicknesses $d$ of the polymersomes measured by Cryo-TEM ${ }^{17}$ were used as the thickness $h$ of the bilayer in the calculation for the polymersome membrane. $R$ was approximated to be close of the radius of gyration $\mathrm{Rg}=\mathrm{N}^{\alpha}(\mathrm{b} / 6)$, where $\mathrm{N}$ is the number of siloxane units, $b$ is the segments' length (Si-O-Si) (= $0.311 \mathrm{~nm})$ and $\alpha$ is the scaling exponent $(=0.66)$ found for membrane thickness of triblock copolymers (ESI of ${ }^{17}$ ). Another approach consists in using the area/chain found in a vesicle to evaluate R. $^{17}$ Table 2 summarizes the membrane characteristics obtained for the different copolymers.

Diffusion coefficients are in good agreement with those reported previously on the PMOXA-b-PDMS-b-PMOXA triblock copolymer, for similar hydrophobic block molar mass. ${ }^{31}$ Interestingly, the membrane viscosity for the grafted copolymer is slightly lower than the membrane viscosity of the triblock $1.5 \mathrm{~K}$, while the molar mass of hydrophobic block is larger. Again, this suggests that copolymer architecture play a role on viscoelastic properties of membrane.

Table 3 Characteristics of membranes obtained from different copolymers and phospholipid POPC (d: hydrophobic core thickness measured by Cryo-TEM, D: lateral diffusion coefficient, $M_{f}$ : mobile fraction and $\eta$ : viscosity of membrane with ${ }^{a}: R$ from $\mathrm{R}_{\mathrm{g}},{ }^{b}: \mathrm{R}$ from area/chain); ${ }^{*}$ bilayer thickness of POPC $\operatorname{Ref}^{43}{ }^{* *}$ with $\mathrm{R}=0.45 \mathrm{~nm}^{44}$.

\section{Giant Hybrid Unilamellar Vesicles}

Regarding stretching elasticity measurements, we will particularly focus on polymer/lipid compositions for which homogenous membrane at the microscale have been reported, but in which lipids nanodomains are present. In order to assess the influence of the presence of lipids in polymersomes on membrane properties, pipette aspiration experiments were performed on GHUVs, which present a relatively extended range of lipid fraction for which homogenous membrane structuration at the microscale has been reported. Such vesicles were obtained with the $3 \mathrm{~K}$ triblock copolymer. ${ }^{1}$ The results obtained are illustrated in Figure 2 .

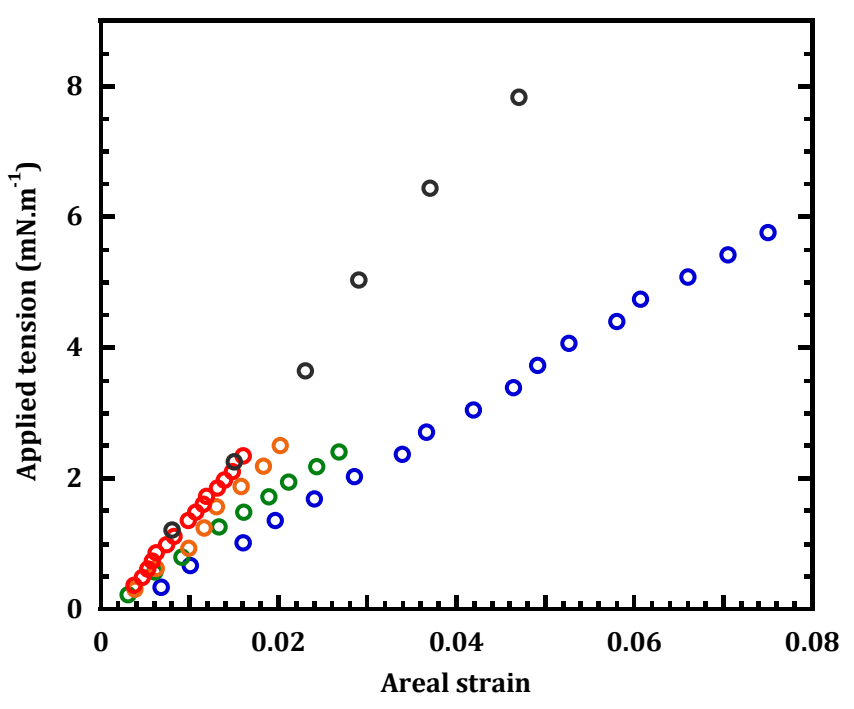

Figure 2. Representative stress - strain curves of 3K/POPC GHUVs at different POPC content: (0): 0\% POPC; (०): 5\% POPC; (०): 10\% POPC (०): 20\% POPC.(\% in w/w) (0): pure POPC (extracted from previous work of the group ${ }^{40}$ ) 
This document is the Accepted Manuscript version of an article that appeared in final form in Soft Matter, 2018, 14(31), 6476-6484, after peer review and technical editing by the publisher. To access the final edited and published work see DOI: 10.1039/C8SM00547H

Interestingly, whereas the few data available reported in literature mention a modulation of stretching moduli and lysis strain in between the value of pure lipid and polymer vesicles, here an increase of stretching moduli is observed when POPC is added, but a drastic decrease of lysis strain is observed even for $5 \% \mathrm{w} / \mathrm{w}$ lipid. The lysis strain obtained are even lower than the lysis strain of pure POPC vesicle ( $5 \%)$, resulting in vesicles presenting a toughness even lower than pure liposomes. This result is quite unexpected, and suggests that if the association of lipids and thicker copolymer chains is effective, as attested by the regular increase of the area expansion modulus (Figure 3 ), instabilities are created probably at the polymer/lipid boundaries. It is worth to recall that the existence of lipid nanodomains has been proven previously by FLIM/FRET experiments. ${ }^{1}$ These nanodomains are in a metastable state and the formation of micrometric lipid domain under tension has been observed for lipid fractions close to those where phase separation occurs at rest $(>30 \% \mathrm{w} / \mathrm{w})$. In our case, before rupture, no micrometric domains have been observed. This suggests that when lipid fractions are low enough, coalescence between lipid domains that allow the decrease of the boundary energy at the lipid/polymer interface is not possible, or is not rapid enough regarding the increase of the line tension at the interface that occurs under pipette aspiration. ${ }^{45}$ This increase of line tension could thus be at the origin of creation of defects in the membrane leading to its premature disruption. Again this effect could be related to the triblock architecture of the copolymer. The behaviour under stretching of hybrid vesicles with a PDMS-g-PEO grafted copolymer, or a PBut-b-PEO diblock copolymer has been studied, although in a restricted range of lipid fraction, and any sign of appearance of embrittlement of the vesicle structure has never been detected, even in the case of a PDMS-g-PEO grafted copolymer, for which hybrid vesicles have been studied by MPA in a composition range where lipid patchy domain were present. ${ }^{24}$

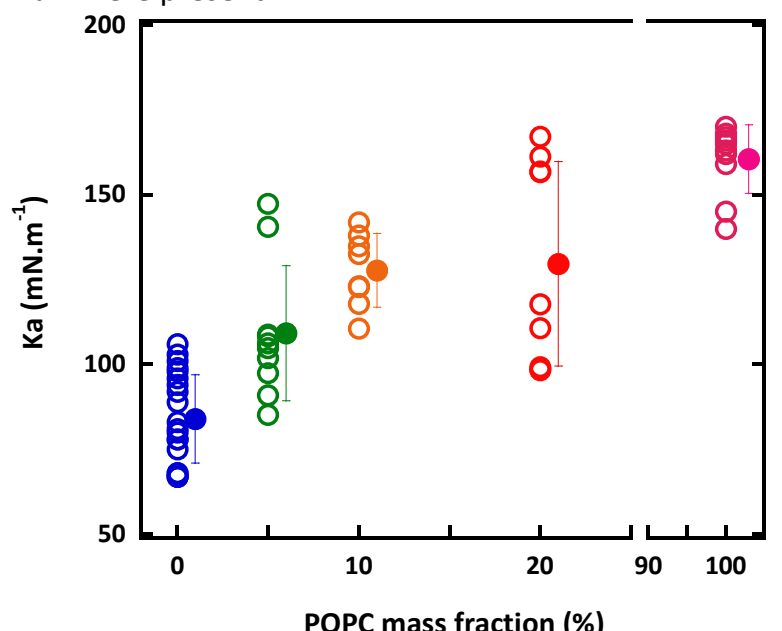

Figure 3 Variation of area expansion modulus of hybrid 3K/POPC GHUVs with POPC content: the open circles are experimental values obtained for individual GHUVs and the closed circles represent the corresponding averaged value with standard deviation (the symbol is slightly shifted to the right for clarity.
The values obtained from different measurements of area expansion modulus are shown in Figure 3-.

It is interesting to note that the dispersity of Ka values is very high. At $20 \%$ of POPC, it seems that two types of vesicles are present in the sample, one with a low Ka, slightly higher than the pure copolymer, and another with significantly higher Ka ( $155 \mathrm{mN} . \mathrm{m}^{-1}$ ). This probably reflects the heterogeneity in vesicle composition due to the fission events commonly observed with 3K/POPC GHUVs. ${ }^{1}$ These events lead to the formation of GHUVs with considerably different membrane compositions.

\section{Lateral diffusion in GHUVs}

Translational diffusion measurements of lipid molecules and copolymer chains were carried out for GHUVs presenting a homogenous and heterogeneous distribution of the component at the microscale. In short, for the $1.5 \mathrm{~K} / \mathrm{POPC}$ mixture, GHUVs with a POPC content of $10 \%, 30 \%$ and $50 \%$ were analysed. Based on our previous study ${ }^{1}$, GHUVs with $10 \%$ POPC are expected to present lipid nanodomains larger than $5 \mathrm{~nm}$ but non-visible under microscopy, whereas samples with $30 \%$ and $50 \%$ POPC show generally visible lipid microdomains. Hence, in FRAP measurements with only polymer fluorescent probe, GHUVs composed of $10 \%$ POPC were seen as homogenous as shown in Figure $\mathrm{S} 4$-a, whereas dark domains were observed in GHUVs composed of $30 \%$ or $50 \%$ POPC (Figure S-4-b) since Dow-FITC does not incorporate in lipid domains at these high lipid fractions. FRAP measurements in these samples were always performed in the polymer-rich phases.

We first tried to evaluate the mobility of polymer chains. The typical FRAP curves for 1.5K/POPC GHUVs at each aforementioned POPC fraction are presented in Figure 4. The effect of POPC on the diffusion of the polymer chains can be qualitatively seen, particularly concerning the mobile fraction. The diffusion coefficients of copolymer chains values versus POPC concentration are represented in Figure 5, and all results are summarized in Tables 4 and 5 .

Diffusion coefficients of polymer molecules in hybrid vesicles seem to be unmodified at a low POPC fraction and then decrease when the POPC fraction increases. The mobile fraction however is slightly modified even at a low POPC content. This variation in diffusivity and mobile fraction is very likely linked to the phase separation occurring in $1.5 \mathrm{~K} / \mathrm{POPC}$ GHUVs. Polymer fluorescent marker Dow-FITC has been shown to be almost completely excluded from lipid phase as illustrated in previous studies. ${ }^{39}$ 
This document is the Accepted Manuscript version of an article that appeared in final form in Soft Matter, 2018, 14(31), 6476-6484, after peer review and technical editing by the publisher. To access the final edited and published work see DOI: 10.1039/C8SM00547H

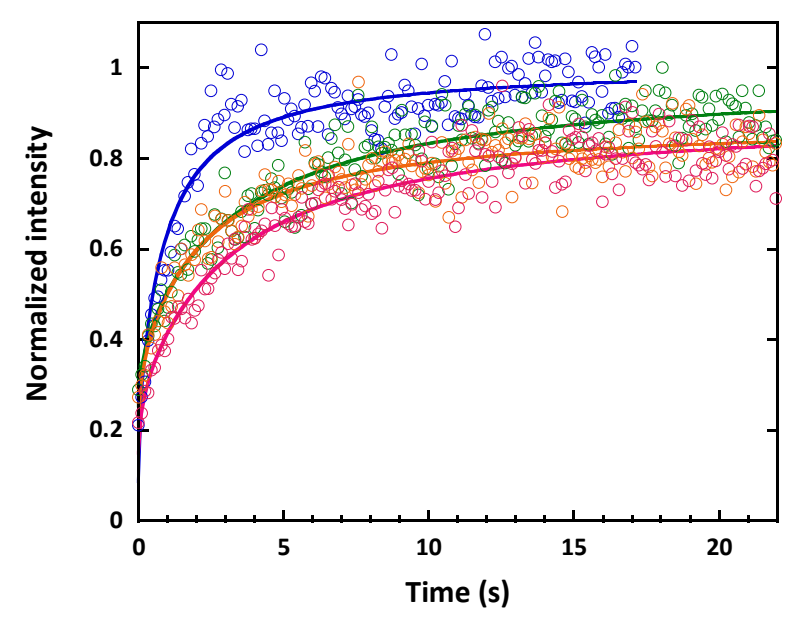

Figure 4 Representative FRAP data obtained from GHUVs with different 1.5K/POPC compositions: (0):0\% POPC; (०):10\% POPC; (०):30\% POPC and (0): 50\% POPC containing $1.5 \%$ molar Dow-FITC. The smooth lines correspond to the fit using the formalisms described in the experimental section.

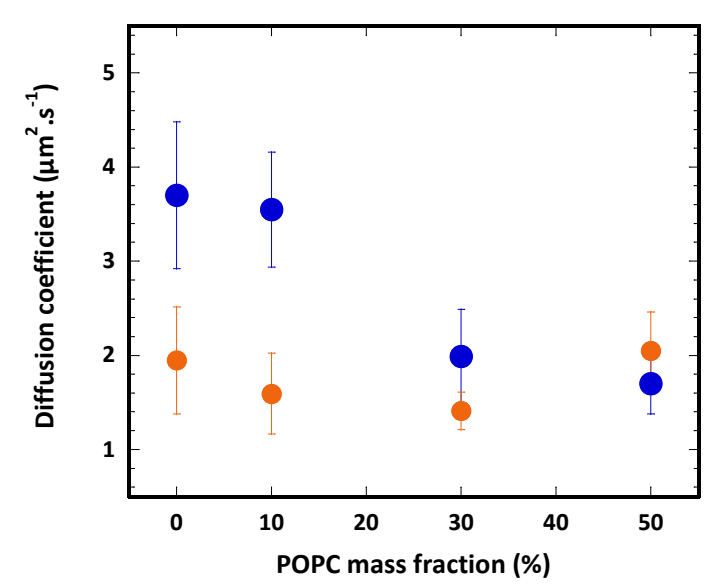

Figure 5 Diffusion coefficients of fluorescent polymer probe in 3K/POPC GHUVs $(\bullet)$ and in $1.5 \mathrm{~K} / \mathrm{POPC}$ GHUVs $(\bullet)$ as a function of POPC content.

Therefore, the diffusion of copolymer chains is hindered due to the presence of these lipid domains. In this case, a small fraction of polymer fluorescent analogue would remain trapped by slower moving nanoscale lipid domains on the bleached ROI (FRAP was always carried out in fluorescent polymer-rich domains). With increasing POPC content, the diffusion coefficients decrease as a result of the presence of more lipid domain obstacles to diffusion.

Similar measurements were carried out for 3K/POPC GHUVs. Interestingly, when POPC content increases, only a slight decrease of diffusion coefficient is observed in this system, as displayed in Figure 5 and Table 5, for GHUVs that do not present phase coexistence under microscope.

In these lipid compositions for the previous block copolymer, variations were more pronounced. This may be due to the higher line tension observed in this system, which decreases the extent of formation of nanoscale lipid inclusions within polymer phases. In the 3K/POPC mixture, the higher line tension promotes fission of lipid domains at significantly higher rates than the ones observed for 1.5K/POPC GHUVs as discussed previously. ${ }^{1}$

\begin{tabular}{cccc}
\hline $\begin{array}{c}\text { POPC } \\
(\%)\end{array}$ & Membrane morphology from ${ }^{1}$ & $\begin{array}{c}\mathrm{D} \pm \mathrm{SD} \\
\left(\mu \mathrm{m}^{2} . \mathrm{s}^{-1}\right)\end{array}$ & $\mathrm{M}_{\mathrm{f}} \pm \mathrm{SD}$ \\
\hline $0 \%$ & - & $3.70 \pm 0.79$ & $\begin{array}{c}1.00 \pm \\
0.01\end{array}$ \\
& & & $0.88 \pm$ \\
$10 \%$ & Nanodomains $>5$ nm but invisible \\
& under microscope & $3.55 \pm 0.61$ & 0.05 \\
& Micro-domains visible under & & $0.82 \pm$ \\
$30 \%$ & microscope & $1.99 \pm 0.50$ & 0.11 \\
& Micro-domains visible under & & $0.82 \pm$ \\
$50 \%$ & microscope & $1.70 \pm 0.32$ & 0.11 \\
\hline
\end{tabular}

Table 4 Membrane morphology and corresponding average lateral diffusion coefficients $(D \pm S D)$ and mobile fractions $\left(M_{f} \pm S D\right)$ of fluorescent polymer analogue in different hybrid 1.5K/POPC membranes.

\begin{tabular}{|c|c|c|c|}
\hline $\begin{array}{l}\text { POPC } \\
(\%)\end{array}$ & Membrane morphology from ${ }^{1}$ & $\begin{array}{c}D \pm S D \\
\left(\mu m^{2} \cdot s^{-1}\right)\end{array}$ & $M_{f} \pm S D$ \\
\hline $0 \%$ & - & $1.95 \pm 0.57$ & $\begin{array}{c}1.00 \pm \\
0.01\end{array}$ \\
\hline $10 \%$ & $\begin{array}{c}\text { Nanodomains }>5 \mathrm{~nm} \text { but invisible } \\
\text { under microscope }\end{array}$ & $1.59 \pm 0.43$ & $\begin{array}{c}0.81 \pm \\
0.08\end{array}$ \\
\hline $30 \%$ & $\begin{array}{c}\text { Nanodomains }>5 \mathrm{~nm} \text { but invisible } \\
\text { under microscope }\end{array}$ & $1.41 \pm 0.20$ & $\begin{array}{c}0.82 \pm \\
0.04\end{array}$ \\
\hline $50 \%$ & $\begin{array}{l}\text { Micro-domains visible under } \\
\text { microscope }\end{array}$ & $2.05 \pm 0.41$ & $\begin{array}{c}0.97 \pm \\
0.07\end{array}$ \\
\hline
\end{tabular}

Table 5. Lateral diffusion coefficient $(D \pm S D)$ and mobile fraction $\left(M_{f} \pm S D\right)$ of fluorescent polymer probe in different hybrid 3K/POPC membranes.

Interestingly, for samples showing micro-domain coexistence under the confocal microscope (3K/POPC GHUVs composed of $50 \%$ POPC), there was no clear difference in both diffusion coefficient and mobile fraction values in comparison with pure polymersomes. This would suggest that there are no more lipid nanodomains in the polymer-rich phase (but there is still some lipid incorporated in the copolymer-rich phase, as Rhod DOPE signal is still detected in confocal images). The explanation for this observation is not obvious. This is probably due to a high line tension at the polymer/lipid boundaries, which drives, above a critical threshold in lipid composition, the existing nanoscale lipid domains to rapidly coalesce into micro-domains. When the lipid composition is close to this critical threshold, micro-domains can also be generated by applying a tension in the membrane as previously shown. ${ }^{1}$ In the other system (1.5K/POPC), nanoscale lipid domains remain 
This document is the Accepted Manuscript version of an article that appeared in final form in Soft Matter, 2018, 14(31), 6476-6484, after peer review and technical editing by the publisher. To access the final edited and published work see DOI: 10.1039/C8SM00547H

trapped within polymer-rich domains probably because of lower line tension.

\section{Lateral diffusion of lipid molecules in hybrid membranes}

The dynamic of lipid molecules in hybrid membranes was also studied through the diffusion coefficient of DOPE-Rhod. This probe partitions preferentially in the lipid phase but not exclusively as shown in previous studies. ${ }^{1,}{ }^{39}$ Figure S5 presents the typical images of $1.5 \mathrm{~K} / \mathrm{POPC}$ GHUVs with $10 \%$ and $30 \%$ POPC (\% wt) respectively. While DOPE-Rhod partitions homogeneously in all the GHUVs at $10 \%$ POPC, it segregated into POPC micro domains in 30\% POPC GHUVs (much brighter phases).

In phase-separated GHUVs, the lipid fluorescent signal is too low in polymer-rich phases to perform FRAP measurements, and the lipid-rich phases are rarely large enough to allow reliable measurements. Therefore, the study was only performed with GHUVs presenting homogeneous membrane structure at the micron scale. Based on the apparent phase diagram, ${ }^{1}$ measurements were carried out with $1.5 \mathrm{~K} / \mathrm{POPC}$ GHUVs containing $10 \%$ and $15 \%$ of POPC. Results are shown in Figure 6.

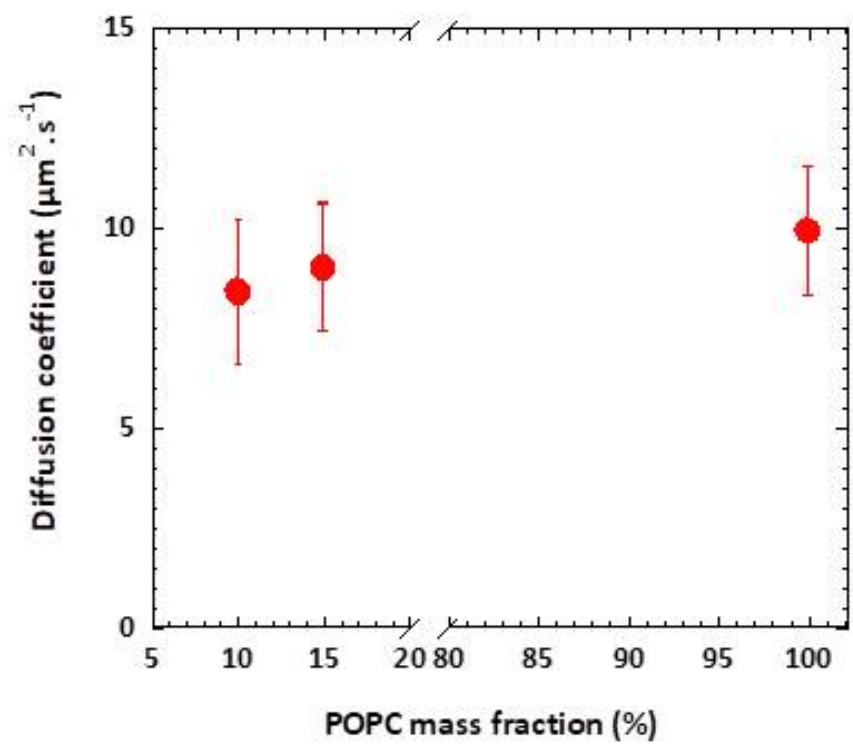

Figure 6. Diffusion coefficients of fluorescent lipid probe DOPE-Rhod in hybrid 1.5K/POPC GHUVs as a function of POPC content.

Lipid molecules diffuse about 2.5 times faster than polymer chains in the same host hybrid membrane $\left(D_{\text {DOPE-Rhod }}=8.4 \pm\right.$ 1.8 vs. $\mathrm{D}_{\mathrm{PDMS}_{26}-\mathrm{g}-\left(\mathrm{PEO}_{12}\right)_{2}}=3.55 \pm 0.61$ in $1.5 \mathrm{~K} / \mathrm{POPC}$ GHUVs with $10 \%$ POPC). Considering the uncertainty of the measurement, there is no difference in diffusion coefficients of lipids in pure POPC and hybrid membranes composed of $10 \%$ and $15 \%$ of POPC. In this way, the diffusion of the lipid analogue is, unlike the copolymer analogue, not affected by nanoscale phase separation. This suggests that FRAP reveals the lateral diffusion of lipid molecules (and not lipid nanodomains which obviously should have a lower diffusion coefficient). Unlike the labelled polymer, the lipids dispersed in the polymer-rich phase can readily exchange with lipids in nanodomains and as such, these domains do not act as barriers for diffusion. This result also suggests that lipid diffusion in hybrid 1.5K/POPC membrane is insensitive to either a vesicle structuration or a composition of hybrid vesicle. This result is different from the observation reported by Vanderlick and col. ${ }^{21}$ in which the lipid lateral- diffusion coefficient in $\mathrm{PBd}_{46}$-b-PEO 30 /POPC GHUVs decreased gradually in proportion to the amount of incorporated polymer. However, since the copolymer used possesses a very low fluidity ( $D=0.22 \pm 0.06 \mu \mathrm{m}^{2} . \mathrm{s}^{-1}-44$ times lower than pure $\mathrm{POPC})$, its influence on the lipid diffusion is probably more pronounced.

\section{Conclusions}

In this work, membrane mechanical properties of GHUVs made from the association of fluid phospholipid (POPC) and triblock copolymer were studied using micropipette aspiration technique. First, the elasticity under stretching of vesicles made from two triblock copolymers with different molar mass were compared to those of well-known grafted commercial copolymers based on the same hydrophobic (PDMS) and hydrophilic (PEO) blocks. The results obtained suggest that the architecture of the block copolymers play a role on membrane toughness, as critical lysis strain was observed to be twice less for triblock copolymers compared to grafted copolymers for the same membrane thickness (or hydrophobic molar mass). Although further studies are needed, evidence obtained from the experiments demonstrated that chain conformation in the polymersome membrane plays an important role on membrane-mechanical properties. Differences in membrane viscosities have been reported in the literature for diblock and triblock copolymers ${ }^{31}$ having the same molar mass, and this was attributed to the presence of a given amount of stretched chain conformation in the case of triblock copolymer. This difference in membrane viscosity has also been observed in this study.

Most importantly, micropipette experiments reveal that the association of triblock copolymer and phospholipids in the same membrane results in a huge decrease of membrane toughness, and leads to the formation of vesicles that are even more fragile than pure liposomes. This was not observed in the few studies available in literature on GHUVs obtained with diblock copolymers. Again, this suggests that the presence of a mixture of hairpin and stretched conformation of polymer chains in the membrane, which is the case for the triblock copolymer, is not really adapted to reach stable edifice in the presence of a low amount of phospholipids. Regarding the mobility of components in hybrid membranes, again the 
This document is the Accepted Manuscript version of an article that appeared in final form in Soft Matter, 2018, 14(31), 6476-6484, after peer review and technical editing by the publisher. To access the final edited and published work see DOI: 10.1039/C8SM00547H

situation is more subtle than in a diffusion simply balanced by the amount of the two components. Polymer chain diffusion is limited by the presence of lipid nanodomains, whereas lipid diffusivity seems to be insensitive to either vesicle structuration or composition of a hybrid vesicle.

Globally, our study shows that the association of polymer and lipid in a single membrane does not lead systematically to "intermediate" properties that could be modulated by the membrane composition. Depending on the architecture of the copolymer used, a great care has to be paid to the characterisation of the membrane structure and the evaluation of hybrid membrane properties. Beyond the fundamental interest of such studies, this is of importance when such structures are considered in biomedical applications. For instance, in drug delivery toughness is important regarding the mechanical stresses that cargos can undergo during blood circulation through vessels. In this still restricted but emerging field of hybrid vesicles/membranes, so far diblock copolymers were most often used, but interest on triblock copolymer architecture is growing. ${ }^{15}$ In this context, it is important that the exact role of copolymer architecture on membrane structuration and properties is deciphered, both at the micro and nanoscale. Further studies on GHUVs and LHUVs using diblock copolymers with same chemical nature (PDMS and PEO) are under way.

\section{Conflicts of interest}

"There are no conflicts to declare".

\section{Acknowledgements}

International doctoral school on Functional Materials (IDSFunMat), Erasmus Mundus (EU), FCT (RECI/CTMPOL/0342/2012, FAPESP/20107/2014, UID/NAN/50024/2013, F.F. is an FCT Researcher - IF/00386/2015)

\section{Notes and references}

1. T. P. T. Dao, F. Fernandes, E. Ibarboure, K. Ferji, M. Prieto, O. Sandre and J.-F. Le Meins, Soft Matter, 2017, 13, 627-637.

2. J. F. Le Meins, C. Schatz, S. Lecommandoux and O. Sandre, Mater. Today, 2013, 16, 397-402.

3. M. Schulz and W. H. Binder, Macromolecular Rapid Communications, 2015, 36, 2031-2041.

4. N. Pippa, D. Stellas, A. Skandalis, S. Pispas, C. Demetzos, M. Libera, A. Marcinkowski and B. Trzebicka, Eur J Pharm Biopharm, 2016, 107, 295-309.

5. N. Pippa, E. Kaditi, S. Pispas and C. Demetzos, Soft Matter, 2013, 9, 4073-4082.
6. N. Pippa, M. Merkouraki, S. Pispas and C. Demetzos, Int J Pharm, 2013, 450, 1-10.

7. O. Bixner, G. Bello, M. Virk, S. Kurzhals, A. Scheberl, N. Gal, A. Matysik, R. Kraut and E. Reimhult, ChemNanoMat, 2016, 2, 1111-1121.

8. Z. Cheng, D. R. Elias, N. P. Kamat, E. Johnston, A. A. Poloukhtine, V. V. Popik, D. A. Hammer and A. Tsourkas, Bioconjugate Chem., 2011, 22, 2021-2029.

9. S. Khan, M. Li, S. P. Muench, L. J. C. Jeuken and P. A. Beales, Chem. Commun., 2016, 52, 11020-11023.

10. W. F. Paxton, P. T. McAninch, K. E. Achyuthan, S. H. R. Shin and H. L. Monteith, Colloids Surf. B, 2017, 159, 268-276.

11. M. Schulz, A. Olubummo, K. Bacia and W. H. Binder, Soft Matter, 2014, 10, 831-839.

12. A. Olubummo, M. Schulz, R. SchÃ fps, J. r. Kressler and W. H. Binder, Langmuir, 2014, 30, 259-267.

13. M. Schulz, S. Werner, K. Bacia and W. H. Binder, Angew Chem Int Ed Engl, 2013, 52, 1829-1833.

14. M. Schulz, A. Olubummo and W. H. Binder, Soft Matter, 2012, 8, 4849-4864.

15. K. Panneerselvam, M. E. Lynge, C. F. Riber, S. MenaHernando, A. A. Smith, K. N. Goldie, A. N. Zelikin and B. Stadler, Biomicrofluidics, 2015, 9, 052610.

16. S. K. Lim, A. S. W. Wong, H.-P. M. de Hoog, P. Rangamani, A. N. Parikh, M. Nallani, S. Sandin and B. Liedberg, Soft Matter, 2017, 13, 1107-1115.

17. T. P. T. Dao, A. Brûlet, F. Fernandes, M. Er-Rafik, K. Ferji, R. Schweins, J. P. Chapel, A. Fedorov, M. Schmutz, M. Prieto, O. Sandre and J. F. Le Meins, Langmuir, 2017, 33, 1705-1715.

18. N. Naziris, N. Pippa, V. Chrysostomou, S. Pispas, C. Demetzos, M. Libera and B. Trzebicka, Journal of Nanoparticle Research, 2017, 19.

19. S. Winzen, M. Bernhardt, D. Schaeffel, A. Koch, M. Kappl, K. Koynov, K. Landfester and A. Kroeger, Soft Matter, 2013, 9, 5883-5890.

20. J. Nam, T. K. Vanderlick and P. A. Beales, Soft Matter, 2012, 8, 7982-7988.

21. J. Nam, P. A. Beales and T. K. Vanderlick, Langmuir, 2011, 27, 1-6.

22. M. Schulz, D. Glatte, A. Meister, P. Scholtysek, A. Kerth, A. Blume, K. Bacia and W. H. Binder, Soft Matter, 2011, 7, 81008110.

23. M. Chemin, P. M. Brun, S. Lecommandoux, O. Sandre and J. F. Le Meins, Soft Matter, 2012, 8, 2867-2874.

24. D. Chen and M. M. Santore, Soft Matter, 2015, 11, 26172626.

25. W. S. Jang, S. C. Park, M. Kim, J. Doh, D. Lee and D. A. Hammer, Macromol Rapid Comm., 2015, 36, 378-384.

26. N. P. Kamat, M. H. Lee, D. Lee and D. A. Hammer, Soft Matter, 2011, 7, 9863-9866.

27. R. Dimova, U. Seifert, B. Pouligny, S. Förster and H. G. Döbereiner, Eur. Phys. J. E, 2002, 7, 241-250.

28. B. M. Discher, Y. Y. Won, D. S. Ege, J. C. M. Lee, F. S. Bates, D. E. Discher and D. A. Hammer, Science, 1999, 284, 1143-1146. 
This document is the Accepted Manuscript version of an article that appeared in final form in Soft Matter, 2018, 14(31), 6476-6484, after peer review and technical editing by the publisher. To access the final edited and published work see DOI: 10.1039/C8SM00547H

29. E. Mabrouk, D. Cuvelier, L. L. Pontani, B. Xu, D. Levy, P. Keller, F. Brochard-Wyart, P. Nassoy and M. H. Li, Soft Matter, 2009, 5, 1870-1878.

30. J. Nam and M. M. Santore, Langmuir, 2007, 23, 7216-7224.

31. F. Itel, M. Chami, A. Najer, S. Loercher, D. Wu, I. A. Dinu and W. Meier, Macromolecules, 2014, 47, 7588-7596.

32. D. Needham and E. Evans, Biochemistry, 1988, 27, 8261-

8269.

33. R. Kwok and E. Evans, Biophys. J., 1981, 35, 637-652.

34. A. Carlsen, N. Glaser, J.-F. Le Meins and S. Lecommandoux, Langmuir, 2011, 27, 4884-4890.

35. Z. Lin, R. M. Hill, H. T. Davis, L. E. Scriven and Y. Talmon, Langmuir, 1994, 10, 1008-1011.

36. R. M. Hill, Langmuir, 1993, 9, 2789-2798.

37. M. I. Angelova and D. S. Dimitrov, Faraday Discussions of the Chemical Society, 1986, 81, 303-311.

38. E. Evans and W. Rawicz, Phys Rev Lett, 1990, 64, 2094-2097.

39. T. P. T. Dao, F. Fernandes, M. Er-Rafik, R. Salva, M. Schmutz, A. Brulet, M. Prieto, O. Sandre and J. F. Le Meins, ACS Macro Letters, 2015, 4, 182-186.

40. T. P. T. Dao, M. Fauquignon, F. Fernandes, E. Ibarboure, A. Vax, M. Prieto and J. F. Le Meins, Colloids Surf. A, 2017, 533, 347-353.

41. H. Bermudez, A. K. Brannan, D. A. Hammer, F. S. Bates and D. E. Discher, Macromolecules, 2002, 35, 8203-8208.

42. P. G. Saffman and M. Delbrück, Proceedings of the National Academy of Sciences of the United States of America, 1975, 72, 3111-3113.

43. N. Kučerka, M.-P. Nieh and J. Katsaras, Biochimica et Biophysica Acta (BBA) - Biomembranes, 2011, 1808, 27612771.

44. F. Itel, M. Chami, A. Najer, S. Lörcher, D. Wu, I. A. Dinu and W. Meier, Macromolecules, 2014, 47, 7588-7596.

45. S. A. Akimov, P. I. Kuzmin, J. Zimmerberg and F. S. Cohen, Phys Rev E Stat Nonlin Soft Matter Phys, 2007, 75, 011919. 